\title{
The Impact of App-Delivered Mindfulness Meditation on Functional Connectivity and Self-Reported Mindfulness Among Health Profession Trainees
}

\author{
Jeremy L. Smith ${ }^{1} \cdot$ Jason W. Allen ${ }^{1,2} \cdot$ Carla Haack $^{3} \cdot$ Kathryn Wehrmeyer $^{4} \cdot$ Kayley Alden $^{4} \cdot$ Maha B. Lund $^{5} \cdot$ \\ Jennifer S. Mascaro ${ }^{4}$ (D)
}

Accepted: 8 September 2020 / Published online: 7 October 2020

(C) Springer Science+Business Media, LLC, part of Springer Nature 2020

\begin{abstract}
Objectives Previous research indicates that mindfulness meditation reduces anxiety and depression and enhances well-being. We examined the impact of app-delivered mindfulness meditation on resting state functional MRI (fMRI) connectivity among physician assistant (PA) students and surgery residents.

Methods PA students and residents were randomized to receive a popular meditation app or to wait-list control group. Before and after the 8-week meditation period, we acquired fMRI scans of participants' resting state, and participants completed a self-report measure of mindfulness. We used a $2 \times 2$, within- and between-group factorial design and leveraged a whole-brain connectome approach to examine changes in within- and between-network connectivity across the entire brain, and to examine whether changes in connectivity were associated with app use or to changes in self-reported mindfulness.

Results Meditation practitioners exhibited significantly stronger connectivity between the frontoparietal network and the left and right nucleus accumbens and between the default mode $(\mathrm{DMN})$ and salience networks, among other regions. Mindfulness practice time was correlated with increased connectivity between the lateral parietal cortex and the supramarginal gyrus, which were also positively correlated with increased scores on the "Describing" subscale of the Five Facet Mindfulness Questionnaire between baseline and post-meditation. These findings are consistent with previous research indicating that mindfulness-based interventions alter functional connectivity within the DMN and between the DMN and other networks both during meditation and at rest, as well as increased connectivity in systems important for emotion and reward.

Conclusions Recent commentaries call for healthcare provider and trainee wellness programs that are sustainable and preventive in nature rather than reactive; these data indicate that even brief sessions of app-delivered mindfulness practice are associated with functional connectivity changes in a dose-dependent manner.
\end{abstract}

Keywords Mindfulness $\cdot$ Meditation $\cdot$ Resting state $\cdot \mathrm{fMRI} \cdot$ Connectivity

Electronic supplementary material The online version of this article (https://doi.org/10.1007/s12671-020-01502-7) contains supplementary material, which is available to authorized users.

Jennifer S. Mascaro

jmascar@emory.edu

1 Department of Radiology and Imaging Sciences, Emory University, Atlanta, GA, USA

2 Department of Neurology, Emory University, Atlanta, GA, USA

3 Department of Surgery, Emory University, Atlanta, GA, USA

4 Department of Family and Preventive Medicine, Emory University, 1841 Clifton Road NE, Suite 507, Atlanta, GA 30329, USA

5 Department of Family and Preventive Medicine, Physician Assistant Program, Emory University, Atlanta, GA, USA
Healthcare profession trainees are at a high risk for depression, burnout, and suicidality (Cocke et al. 2019; Mata et al. 2015; Rotenstein et al. 2016). While causes are multi-factorial, providing resources to help increase resilience of trainees is imperative, and several studies indicate that the cultivation of mindfulness may buffer trainees from the harmful effects of intensive medical training (Chaukos et al. 2017; McConville et al. 2017). While early research on mindfulness meditation was beset with methodological flaws (Ospina et al. 2008), more recent rigor and systematic reviews indicate that mindfulness enhances well-being (Demarzo et al. 2015), reduces anxiety and depression (Goyal et al. 2014), and optimizes immune physiology (Black and Slavich 2016) and daily functioning (Neuendorf et al. 2015). Medical training programs 
have taken note and several medical schools currently offer mindfulness courses, with curricula ranging from lectures to multi-week programs (Dobkin and Hutchinson 2013). Accumulating research indicates that mindfulness meditation reduces anxiety and depression and enhances well-being among health profession trainees, and a recent meta-analysis highlighted the potential efficacy of mind-body wellness programs such as mindfulness meditation for improving medical trainees' well-being (Lomas et al. 2018; McConville et al. 2017).

While mindfulness interventions are promising for augmenting resilience, to date the majority of studies examining mindfulness among health professionals have examined timeintensive interventions that are prohibitive for the majority of trainees (however, see Lebares et al. 2018). Most of these studies have used Mindfulness-Based Stress Reduction (MBSR) interventions, which traditionally involve an extensive time commitment. Mindfulness training has become more feasible with the development and popularity of mobile application (app)-delivered meditation content. While several recent studies suggest that app-delivered mindfulness meditation reduces self-reported stress, irritability (Economides et al. 2018), depression (Howells et al. 2016), burnout, and compassion fatigue (Wylde et al. 2017), and attenuates the cortisol response to a psychosocial stress test (Lindsay et al. 2018), few studies have examined the potential for using appdelivered mindfulness for health profession trainees. Moreover, the existing research on mindfulness for trainees has almost exclusively relied on self-report assessments to evaluate outcomes (Economides et al. 2018). Few studies have examined the impact of mindfulness on trainee brain function (however, see Lebares et al. 2019), and to our knowledge, no studies have examined the impact of mindfulness on functional connectivity in health profession trainees.

Emerging evidence indicates that health-relevant effects of mindfulness are mediated by alterations to the default mode network (DMN), to the salience network (SN), and to the systems involved in executive control often referred to as the central executive network (CEN) (Creswell et al. 2016; Mooneyham et al. 2016). Four related lines of research provide support for the role of the DMN and $\mathrm{SN}$ in particular as key neural mechanisms for the cultivation of mindfulness. First, studies indicate that mindfulness meditation in the fMRI scanner recruits these networks (Brewer et al. 2011; Farb et al. 2012; Hasenkamp et al. 2012; Kilpatrick et al. 2011). Second, mindfulness meditation training has been shown to alter intrinsic functional connectivity within and between these networks during rest in both healthy (Brewer et al. 2011; Jang et al. 2011; Taylor et al. 2012) and clinical populations (Wells et al. 2013). Third, self-reported trait mindfulness scores are related to intrinsic functional connectivity within and between these networks at rest (Bilevicius et al. 2018; Doll et al. 2015). Fourth, studies have demonstrated that mindfulness training impacts functional activity in or connectivity within and between these networks during tasks such as reappraisal or induction tasks (see Gotink et al. 2016).

While the majority of studies to date have focused on changes in connectivity within and between the DMN, SN, and CEN networks, some research indicates that mindfulness training and practice induce connectivity changes in other networks. For example, at least three studies have found that mindfulness training (Doll et al. 2016; Hölzel et al. 2013) or dispositional mindfulness (Modinos et al. 2010) were related to increased functional connectivity between the PFC and the amygdala. These findings are generally interpreted as an increase in emotion regulation via prefrontal control of the amygdala, an interpretation that is consistent with studies finding reduced amygdala reactivity with mindfulness training (Farb et al. 2007).

For this study, we use a longitudinal and randomized waitlist controlled design to examine the impact of app-delivered mindfulness meditation on resting state functional connectivity among physician assistant students and surgery residents. In contrast to other studies that focused on the DMN, SN, and CEN using seed-based connectivity approaches, we used a $2 \times 2$, within- and between-group factorial design and a whole-brain connectome approach to query within- and between-network connectivity across the entire brain. Based on prior studies, we hypothesized that app-delivered mindfulness is effective for health professions trainees; that this efficacy is mediated by changes in DMN, SN, and CEN network connectivity; and that these changes in connectivity would be positively associated with practice time and with changes in self-reported trait mindfulness.

\section{Methods}

\section{Participants}

Surgery residents $(n=9)$ and physician assistant (PA) students $(n=16)$ were recruited via in-person presentations held after class or grand rounds. Upon providing informed consent in accordance with the University's Institutional Review Board, participants were randomly assigned using a random number generator to either a mindfulness meditation program using the 10\% Happier application (practitioners group), and were asked to practice app-guided mindfulness meditation for approximately $12 \mathrm{~min} /$ day for 8 weeks, or to a wait-list (control group). Prior to randomization and again after completion of the 8-week program, participants completed self-report assessment and fMRI described in detail below. Three participants were removed from analysis for excessive movement during fMRI, resulting in a cohort of 13 practitioners $(10$ [77\%] women) and 9 controls (7 [78\%] women) (Table 1). 
Table 1 Demographic information for all participants

\begin{tabular}{llll}
\hline & App & WL & $p$ value \\
\hline Sex/gender & $10 \mathrm{~F}, 3 \mathrm{M}$ & $7 \mathrm{~F}, 2 \mathrm{M}$ & 0.96 \\
Race & & & 0.53 \\
American Indian & 0 & 0 & \\
Native Hawaiian or other Pacific Islander & 0 & 0 & \\
White & 10 & 7 & \\
African, African-American & 1 & 0 & \\
Asian & 1 & 2 & 0.25 \\
Other & 1 & 0 & 0.98 \\
Trainee position & $6 \mathrm{R}, 7 \mathrm{PA}$ & $2 \mathrm{R}, 7 \mathrm{PA}$ & $125.8(20.1)$ \\
Time 1 FFMQ Sum & $125.6(22.1)$ & $22.6(5.73)$ & 0.39 \\
Awareness & $25.1(7.12)$ & $24.9(8.62)$ & 0.68 \\
Non-judgment & $26.2(6.47)$ & $22.3(4.27)$ & 0.92 \\
Non-reactive & $22.5(5.27)$ & $27.3(6.08)$ & 0.59 \\
Describing & $26.0(5.39)$ & $28.7(4.69)$ & 0.19 \\
Observing & $25.8(5.02)$ & & \\
\hline
\end{tabular}

App assigned to mindfulness application group, $W L$ assigned to wait list group, $F$ female, $M$ male, $R$ residents, $P A$ physician assistant students, $F F M Q$ Five-Factor Mindfulness Questionnaire
Participants were compensated $\$ 100$ for completing both assessments. This study was pre-registered (NCT03452670) with self-reported incivility as the primary outcome measure, which will be examined at the level of the entire trainee group and will be reported in a subsequent manuscript.

\section{Procedure}

Participants randomized to the practitioner group were provided a 1-year subscription to the app 10\% Happier (https://www. tenpercent.com). We chose $10 \%$ Happier among a variety of meditation apps that are currently on the market given its orientation toward practical applications of mindfulness and its marketing toward "fidgety skeptics" (Harris et al. 2018), which we thought would appeal to busy trainees. For example, the app notes, "Just in case you're worried, meditation does not require a lot of the things people think it might. For example, you don't have to sit in a particular position. (Unless you want to, of course.) You also don't have to light incense, chant, or believe in anything in particular. There's nothing to join, no special outfits to wear." (tenpercent.com 2020) The app company provided subscriptions for the study participants, suggestions on recommended content, and anonymized app usage data. App usage was acquired from 10\% Happier and is reported as the elapsed time (in minutes) that the app meditation modules were used by each person.

Participants were given the following instructions: "We would like for you to try to practice every day for 8 weeks, even if it is only for one minute. Based on mindfulness research and on the suggestions of the app developers, we would like you to try the following programs: 'The Basics' and
'Emotional Agility.' If you are pressed for time and cannot do a module from the programs, please do the 'One minute counts'." The Basics program included 16 modules with didactic instructions and mindfulness practice time varying between 4:20 to 13:22 (average meditation length: 9:44). This program is an introduction to mindfulness meditation taught by a prominent meditation instructor (Joseph Goldstein), and the practices include mindfulness to the sensations of the breath, mindfulness toward sensations in and experiences of the body, and mindfulness toward the contents of the mind. In addition, the meditations encourage the practitioner to use the skill of "mental noting" to label their mental contents. The "Emotional Agility" program included 15 modules with didactic instructions and mindfulness toward mental content (with a focus on emotions). Meditation practice in the "Emotional Agility" program varied from 11:00 to 13:00 (average meditation length: 12:18). The practices in these modules also include mindfulness toward the sensations of the breath and body, but further aim to cultivate awareness and understanding of emotions as mental contents and the nonjudgmental stance toward emotions with a goal of optimizing the response to one's emotions.

\section{Measures}

\section{Self-Reported Mindfulness}

To evaluate whether app-delivered mindfulness impacts selfreported mindfulness, we administered the Five Facet Mindfulness Questionnaire (FFMQ: Baer et al. 2004, 2008) to all participants prior to randomization and upon completion 
of 8 weeks of mindfulness practice. The FFMQ is a 39-item instrument that uses a 5-point Likert-type scale $(1=$ never or rarely true, $5=$ very often or always true). The FFMQ measures self-reported trait scores on five subscales thought to be components of mindfulness in everyday life: act with awareness (e.g., "When I do things, my mind wanders off and I'm easily distracted"), non-judgment of one's inner experience (e.g., "I criticize myself for having irrational or inappropriate emotions"), non-reactivity to one's inner experience (e.g., "I perceive my feelings and emotions without having to react to them"), Describing (e.g., "I'm good at finding words to describe my feelings"), and observing (e.g., "When I'm walking, I deliberately notice the sensations of my body moving"). Changes in self-reported mindfulness were evaluated by repeated measures ANOVA in SPSS (Version 25).

\section{fMRI}

Baseline and post-program resting state functional MRI (rsMRI) data were acquired on a 3 T Siemens Prisma FIT (8-min multiband acquisition with $2 \mathrm{~s}$ repetition time, $2.97 \times 2.97 \times 2.00 \mathrm{~mm}$ voxels, $70^{\circ}$ flip angle, echo train length $=37$ ). All preprocessing and bivariate correlation (connectome) analyses were performed in the CONN Toolbox (Whitfield-Gabrieli and Nieto-Castanon 2012). Preprocessing of the rsMRI and anatomical volumes in CONN, which wraps SPM8 (www.fil.ion.ucl.ac.uk/spm) and aCompCor (Behzadi et al. 2007) noise source removal functions, consisted of slice timing, field-map, and motion correction; rsMRI/anatomical/MNI standard space coregistration and normalization; smoothing at $5 \mathrm{~mm}$ filter width at half-maximum, linear detrending, bandpass filtering at $8-90 \mathrm{mHz}$, and regression of the six motion parameters and their first-order derivatives, as well as CSF and white-matter signals (Birn et al. 2006; Fox et al. 2005, 2009), by general linear model (GLM). We did not take the controversial step of adding the mean whole-brain signal as a regressor, as there is some evidence that doing so may artificially introduce negative correlations, and that the aCompCor method, in combination with bandpass filtering and orthogonalization of motion parameters, is preferable to global signal regression (Chai et al. 2012). All structural and denoised functional data, gray matter, white matter, and CSF masks were manually inspected to confirm registration validity. Additionally, a Fisher (inverse hyperbolic tangent) transformation was applied to bivariate correlation measures prior to between-subjects analysis in order to meet the normality assumptions of the general linear model. Subjects were normalized (registered) to the Montreal Neurological Institute (MNI) template via SPM's nonlinear, diffeomorphic method (DARTEL), which afforded both forward and backward transformations between subject and template space (Ashburner 2007; Ashburner and Friston 2011). Regions of interest (ROI) were predefined in the CONN Toolbox and are largely derived from the AAL atlas and FSL Harvard-Oxford Maximum Likelihood Cortical and Subcortical atlases (Desikan et al. 2006; Frazier et al. 2005; Makris et al. 2006; TzourioMazoyer et al. 2002).

\section{Data Analysis}

A whole-brain connectivity matrix, representing the correlation between any two pairs of ROIs after accounting for the mediating effects of all other ROIs, was then computed for each subject. Each cell in the connectivity matrix contains a multivariate regression coefficient, or beta weight, estimated from a multiple regression model using the correlation between the two ROIs as the dependent variable and their correlation with all other ROIs designated as the predictor matrix. The beta weights for a given pair of ROIs can be extracted across subjects and correlated with a variable, such as a cognitive score, to obtain a measure of the degree of psychophysiological interaction (PPI). For the present study, these PPI variables included FFMQDescribing scores, practice time, and treatment (practitioners vs. controls). However, as we were interested in the difference in FFMQ-Describing scores between the baseline and post-program time points, we computed $\triangle F F M Q($ Des $)=F F M Q(D e s)_{\text {post }}-F F M Q(\text { Des })_{\text {pre }}$ as a surrogate variable. Three PPI analyses were conducted: one for the main effect of treatment at baseline and follow-up, one for the treatment $\times \triangle F F M Q(D e s)$ interaction, and one for the treatment $\times \triangle F F M Q(D e s) \times$ practice time interaction.

A threshold of $p \leq 0.05$ was applied to all analyses with false discovery rate (FDR) correction for multiple comparisons. The voxelwise connectivity matrix was corrected for topological false discovery rate (Worsley et al. 1996) and reduced to a region of interest (ROI)-to-ROI connectivity matrix defined by a priori atlas regions from the Automated Anatomical Labeling (AAL) atlas, FSL Harvard-Oxford Maximum Likelihood Cortical and Subcortical atlases, and Thalamic Deep-Brain Stimulation (DBS) Connectivity atlas. An F-statistic was computed for each ROI pair via multivariate parametric general linear model, yielding an uncorrected cluster-level $p$ value $<0.05$ (the likelihood of a randomly selected ROI pair showing equal or larger effects) and a clusterlevel $p$ value corrected for false discovery rate (Benjamini and Hochberg 1995; Jafri et al. 2008).

Regression and spectral density analyses were performed in Alteryx Designer (v2019.1) and via in-house Python code, respectively. Power spectral density was calculated using Welch's method in SciPy (v1.4.1) with segment length = $240, F_{s}=0.50 \mathrm{~Hz}$, overlap 120 . 


\section{Results}

Participants randomized to the practitioner group used the app between 0 and 466.2 min (mean $=193.9 ; \mathrm{SD}=161.2$ ). Two trainees (both PA students) randomized to the practitioner group did not use the app at all, but were still included in the intent-to-treat analysis. There was no significant difference between the surgery residents $($ mean $=206.8 ; \mathrm{SD}=147.9$ ) and PA students (mean $=182.8 ; \mathrm{SD}=182.8$ ) in terms of practice time $\left(t_{11}=0.26, p=0.80\right)$. There was a significant group by time interaction for the "Describing" subscale of the FFMQ, such that practitioners reported increased scores compared with those randomized to the waitlist group $[F(20)=$ $\left.7.28, p=0.014, \eta_{\mathrm{p}}{ }^{2}=0.27\right]$. No other subscales of the FFMQ were significant for a group by time interaction. Changes in "Describing" were significantly correlated with mindfulness practice time within the practitioner group (Spearman $r_{\mathrm{s}}=$ $0.66, p=0.013$ ).

An initial set of analyses failed to reveal significant differences in region-to-region (ROI-ROI) connectivity between the control and practitioner groups on the baseline MRI. With respect to the follow up 8-week visit (after completion of meditation training in the practitioner group), two networks, one with a right lateral parietal hub (LP, default-mode network) and one with a left posterior parietal hub (PPC, frontoparietal network), differed significantly between controls and practitioners. LP network vertices were comprised of the left and right supramarginal gyri (SMG, salience network), left and right lateral visual cortices, left inferior frontal gyrus, and the posterior aspect of the left superior temporal gyrus (pSTG); PPC network vertices consisted of the left and right accumbens nuclei (Fig. 1a). In all cases, practitioners exhibited stronger connectivity between the hubs and vertices relative to controls [minimum $t$ (practitioners $>$ controls $)=+$ $\left.3.45 \sim p_{F D R}=0.0194\right]$. Only the right LP network (specifically, the left and right supramarginal vertices) attained significance for the treatment $\times \triangle F F M Q(D e s)$ interaction. The supramarginal vertices of the right LP network also attained significance for the treatment $\times \triangle F F M Q($ Des $) \times$ practice time interaction (see Figs. $1 \mathrm{~b}$ and 2). The PPC network failed to attain significance for both the treatment $\times \triangle F F M Q($ Des $)$ and treatment $\times \triangle F F M Q(D e s) \times$ practice time interactions. We also noted that these effects were driven primarily by the PA students, likely due to too few residents randomized to the control group, and performed a second set of analyses based solely on these subjects which are provided in the Supplementary Materials.

These findings suggest that the functional connectivity of the right LP/SMG network is positively correlated with greater differences in $F F M Q(D e s)$ and that modulation of connectivity within this network increased with mindfulness training. The strength of these connections increased proportionally with longer practice times (see Fig. 2). Both $\triangle F F M Q(D e s)$ (across both control and practitioner groups) and practice time (within the practitioner group) were reliable predictors of lateral parietal/supramarginal connectivity under linear regression, with $\triangle F F M Q(D e s) R^{2}=20.85 \%$ for R LP:L SMG and $22.48 \%$ for R LP:R SMG, and practice time $R^{2}=15.72 \%$ for R LP:L SMG and $22.49 \%$ for R LP:R SMG (Fig. 3).

Practitioners exhibited differences in right LP and SMG spectral power at several frequencies at +8 weeks relative to baseline and to control subjects at +8 weeks, particularly at frequencies $<6 \mathrm{mHz}$ (Fig. 4a). Moreover, practitioners exhibited greater shared power (cross-spectral density) between the right LP hub and the SMG vertices at these frequencies, compared with both baseline and with control subjects at +8 weeks, suggesting that the increase in connectivity observed in practitioners' time-domain data may be due to a reduction in signal "noise," an increase in signal coherence, or a combination. Our connectivity and frequency-domain results are provided in tabular form as Tables 2 and 3, respectively. Again, results for the PA students only are provided in the Supplemental results.

\section{Discussion}

Here we tested the hypothesis that healthcare profession trainees randomized to use a popular mindfulness meditation app would display alterations in functional connectivity, and that these changes would be associated with both practice time and with increases in facets of self-reported trait mindfulness. We found that trainees randomized to mindfulness meditation had increased self-reported Describing scores, a change that was significantly correlated with practice time. In addition, trainees randomized to mindfulness had increased functional connectivity between the CEN and the nucleus accumbens and between nodes of the DMN and SN. Changes in functional connectivity were related to both subjective (self-reported Describing) and objective (app usage data) meditation outcomes, and to our knowledge, this is the first study to report such effects.

Interestingly, $\beta$ weights from the regression analyses indicate that mindfulness practice time might be less predictive of changes in connectivity than changes in self-reported mindfulness. Others have noted the problematic nature of selfreport measures of mindfulness and argued that self-report assessment tools may not adequately measure changes that occur during mindfulness training (Lutz et al. 2015; Van Dam et al. 2012). While our findings do not mitigate these concerns and we interpret them with warranted caution, the fact that changes in facets of self-reported mindfulness, namely Describing, were more closely related to changes in brain function may reflect variance across participants in terms of the quality or effectiveness of practice time. Practice time in the current study was measured objectively via app usage data 
(a) $\quad \begin{array}{lll}-5 & \mathbf{t} & +5\end{array}$

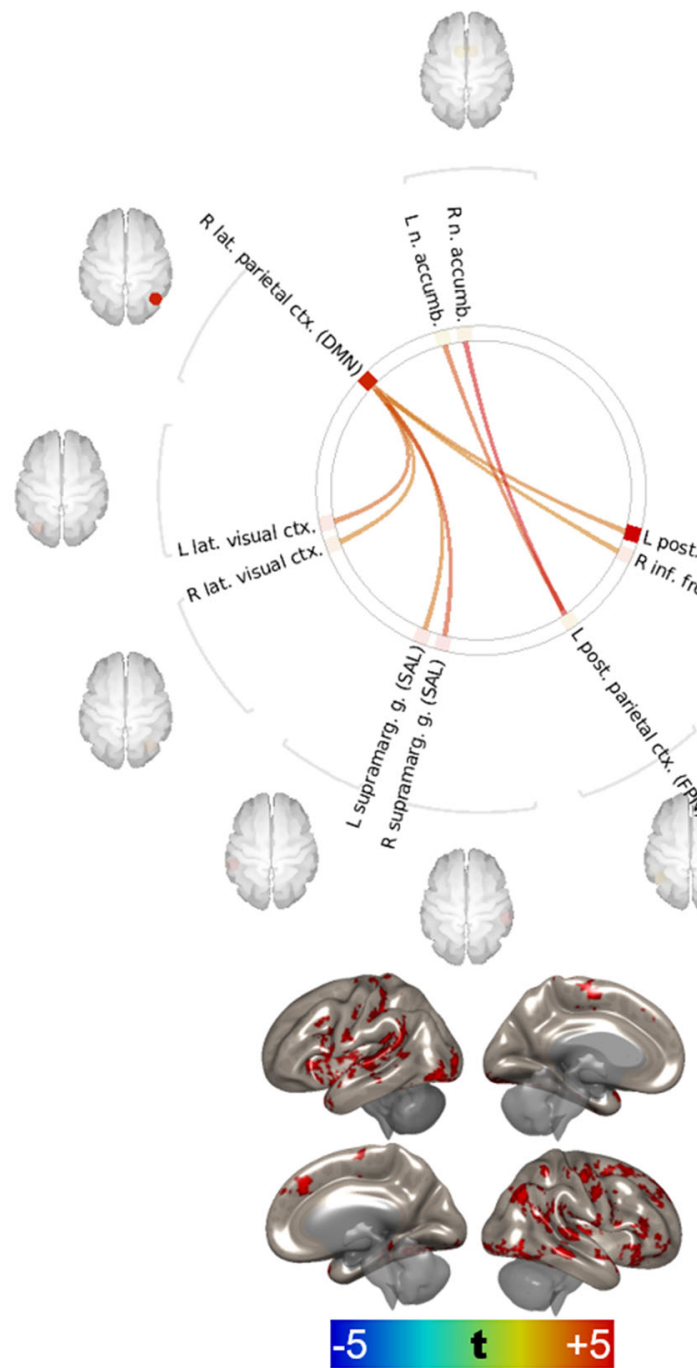

(b) $\begin{array}{lll}-5 & \text { t } & +5\end{array}$
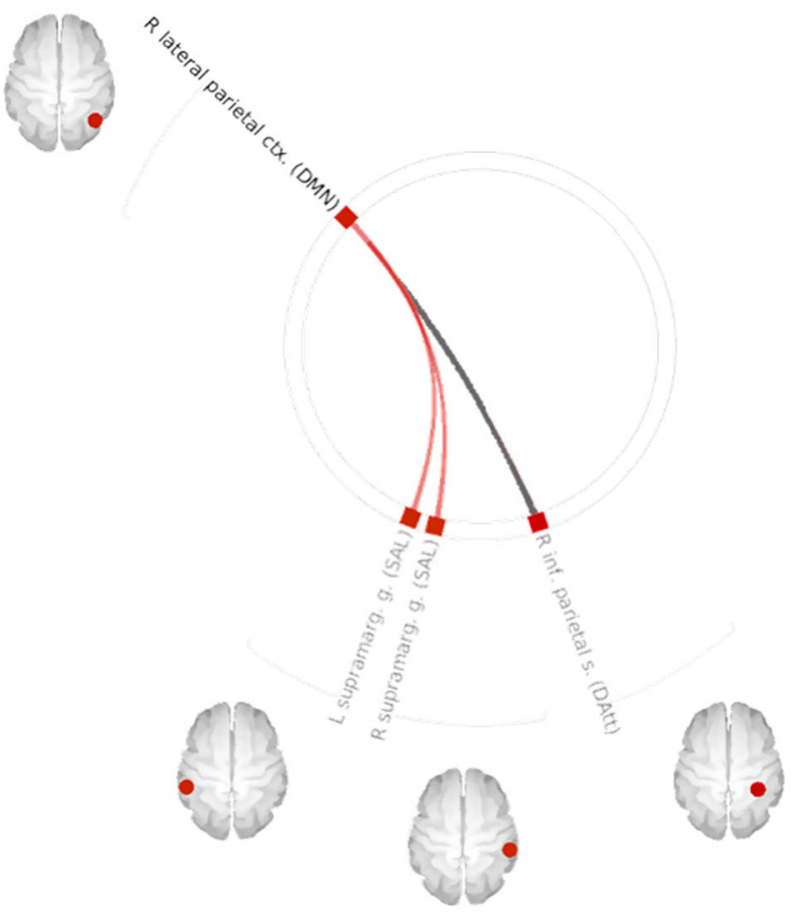

Fig. 1 a Comparison of the effect of treatment (practitioners versus controls) at +8 week-follow-up visit. Warm colors represent stronger connectivity (higher ROI-ROI correlations) in practitioners than controls at +8 weeks; cool colors, weaker connectivity (lower ROI-ROI correlations) in practitioners than controls at +8 weeks. The effect of treatment on the connectivity of right lateral parietal and left posterior parietal cortices is presented below the carousel plot. $t$ test; FDR correction by

and thus was likely more accurate than self-reported practice time. Nonetheless, similar findings were found in functional connectivity alterations when correlating either practice time or self-report Describing, providing corroborative evidence of the importance of these regions in underlying changes associated with mindfulness meditation.

Because the observed impact of mindfulness training was on networks in a resting state rather than active during a task, we cannot definitively link these changes with cognitive outcomes, processes, or mechanisms. However, these findings are consistent with findings from several previous studies of the impact of longer mindfulness programs on brain function.
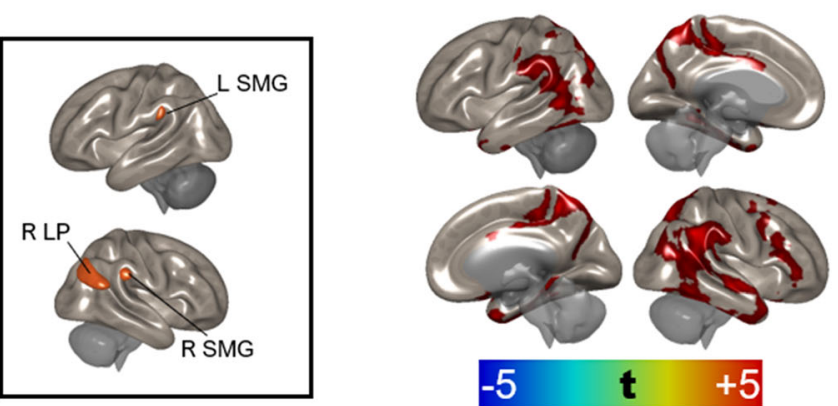

Benjamini-Hochberg method. b Effect of $\triangle F F M Q(D e s)$ on connectivity at +8 weeks over all subjects (practitioners and controls). Warm colors indicate increased connectivity (ROI-ROI correlations) with greater increases in $\operatorname{FFMQ}(D e s)$ from baseline to +8 weeks. The connection in gray was not significant for the treatment effect shown in Fig. 1a. The effect of $\triangle \mathrm{FFMQ(Des)}$ on right lateral parietal connectivity is shown below the carousel plot. FDR correction by Benjamini-Hochberg method

Overall, the current study identified changes in connectivity within and between the DMN, CEN, and SN, results consistent with previous research and with theoretical accounts of the role of these networks in mindful attention. The CEN typically becomes active during cognitively demanding tasks, such as those requiring working memory or decision-making and goal-directed judgments (Koechlin and Summerfield 2007; Petrides 2005). In contrast, the DMN typically becomes active during task-negative periods of resting state, and the network has been associated with self-referential processing, autobiographical and episodic memory, and mind-wandering (Buckner and DiNicola 2019). Crucially, the CEN and the 
Fig. 2 a Effect of practice time on connectivity at +8 weeks in practitioners only. Warm colors indicate stronger connectivity (ROI-ROI correlations) with greater practice time over the 8week experiment. b Connectivity of right lateral parietal cortex as effect of practice time in practitioners. FDR correction by Benjamini-Hochberg method (a) $\begin{array}{lll}-5 & \mathbf{t}+5\end{array}$

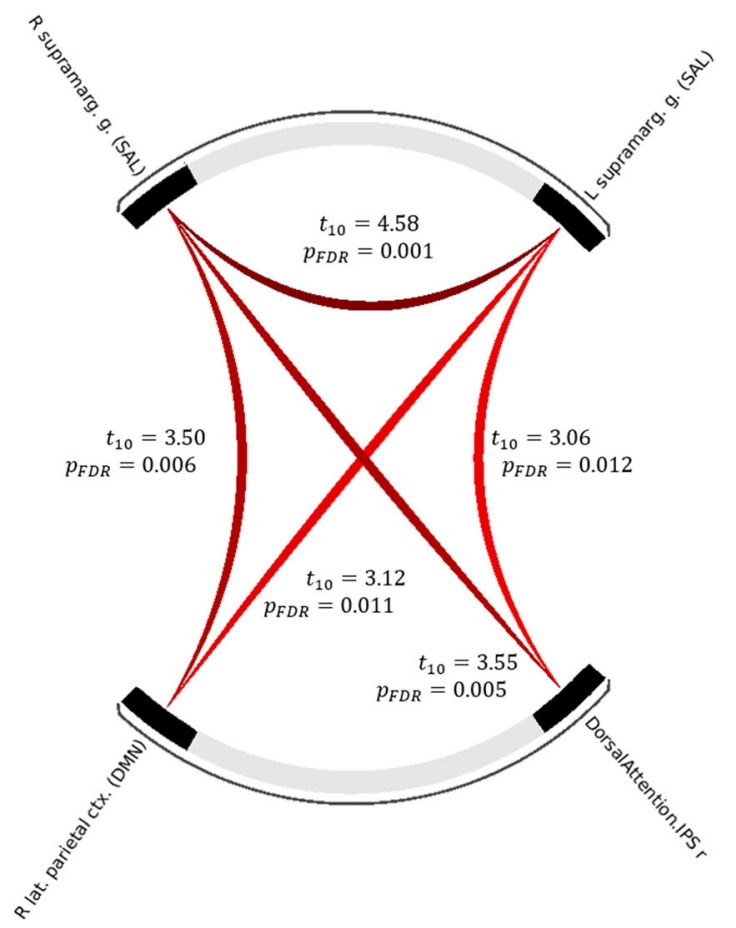

(b) $\begin{array}{lll}-9 & \mathbf{t} & +9\end{array}$

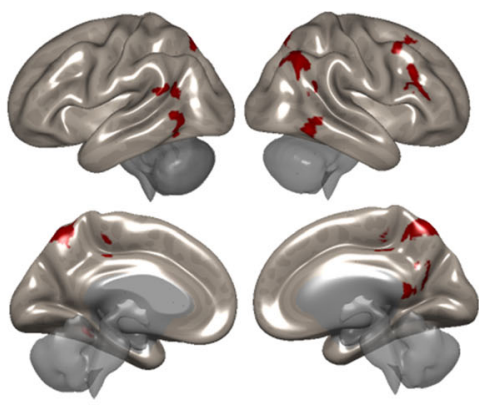

DMN are often anti-correlated and appear to be intrinsically oppositional to one another (Kelly et al. 2008). The triple network hypothesis posits that the DMN and the SN interact with the CEN for effective detection, integration, and response in the context of internal and external events. Fundamental to this model is that the anterior insula is a hub of the SN that deploys the executive control network for sustained attention and response selection toward salient stimuli (Menon and Uddin 2010).

Changes in connectivity between the right lateral parietal (DMN) and left supramarginal gyrus (SN) were associated with both practice time and with scores on the Describing subscale of the FFMQ. One study found that the describing aspect of mindfulness, as measured by the FFMQ, was associated with gray matter volume in the anterior insula and amygdala, a finding that they interpreted as indicative of these regions being important for integrating interoceptive awareness to report on one's emotional states (Murakami et al. 2012). Another study found that mindfulness training among elite athletes increased activity in the anterior cingulate cortex while athletes anticipated an interoceptive challenge, and that this increased activity was positively associated with the "Describing" subscale of the FFMQ (Haase et al. 2015).

While the most prominent hubs of the SN are the dorsal anterior cingulate cortex and anterior insula (Seeley et al. 2007), the supramarginal gyrus may be included in this network (Wang et al. 2017) and has been shown to covary with salient stimuli on task fMRI (Downar et al. 2002). The study referenced above reported that elite athletes had increased activation in the left supramarginal gyrus during an interoceptive challenge, but this activation was not related to trait mindfulness scores (Haase et al. 2015). Furthermore, a separate study found trait mindfulness (using the Mindful Attention Awareness Scale) was correlated with functional activity in the supramarginal gyrus/temporoparietal junction during focused breathing compared with mind-wandering (Dickenson et al. 2012). The authors interpreted this activity as broadly consistent with this region's role in shifting attention back to a target following distraction. While participants in our study were not given any explicit instructions in the scanner, a germinal study found that meditation adepts had increased functional connectivity patterns that were similar both at rest and during meditation, and they interpreted this findings as indicating that meditators may be "on task" even during the resting state (Brewer et al. 2011). Another study found that individuals randomized to a mindfulness induction had more activity in the left supramarginal gyrus while anticipating a negative emotional stimulus (Lutz et al. 2013).

While these results are consistent with a wealth of previous research, there is some contradiction in the existing research as to whether mindfulness increases or decreases DMN connectivity. Some of these contradictions, however, may be partially explained by differences in methodological approach and the definition of connectivity. Brewer et al., for example, have interpreted smaller fMRI signal amplitudes in the defaultmode medial prefrontal (mPFC) and posterior cingulate 


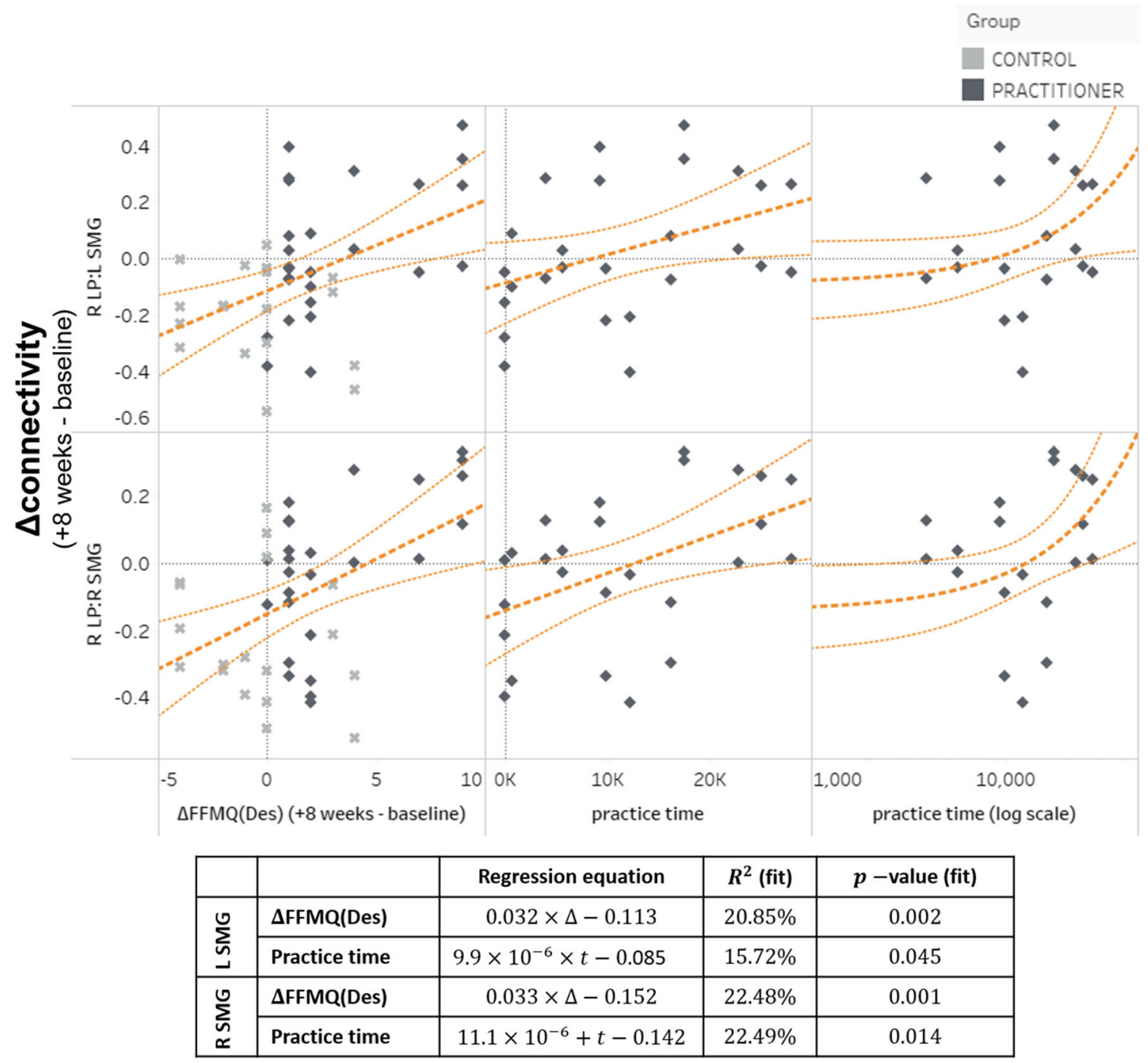

Fig. 3 Changes in connectivity between the default-mode (right hemisphere lateral parietal cortex) and salience networks (supramarginal gyri) as a function of either a difference FFMQ(Des) scores between visit 2 and visit 1 (left column) or time spent practicing with the meditation application (right column). Regression line is in dark orange; thin lines represent $95 \%$ confidence intervals. Overall significance of regression line fits is shown in the table

suggest that the strength of correlations within the DMN, and between the DMN, SN, and CEN, may increase as a function of meditation practice despite this attenuation. However, ascription of these changes in correlations to particular neural or neuronal processes is complicated by the fact that the correlation measure itself is sensitive to phase differences between the regions and to the presence of noise (random noise and fluctuations of no interest) in the signal. We attempted to address this issue with frequency-domain, or spectral, analyses, which are more robust to the presence of uncorrelated noise and to the phase differences between the brain regions (Duff et al. 2008). Our spectral density plotsessentially histograms of the number of data points occurring at a given frequency - indicated a broad reduction in spectral power in practitioners, but not controls, which suggests that the stronger correlations observed in practitioners were due to 
Table 2 Main effect of treatment (see Fig. 1a) and interaction effects [treatment $\times F F M Q($ Des $)($ Fig. 1b) and treatment $\times F F M Q($ Des $) \times$ practice $($ Fig. 2)] were conducted consecutively

\begin{tabular}{|c|c|c|c|c|c|c|c|}
\hline & \multirow[b]{2}{*}{ Destination ROI } & \multicolumn{2}{|c|}{ Treatment } & \multicolumn{2}{|c|}{$\times \Delta$ FFMQ(Des) } & \multicolumn{2}{|c|}{$\times$ PRACTICE } \\
\hline & & $\mathrm{t}(20)$ & $\mathrm{p}(\mathrm{FDR})$ & $\mathrm{t}(20)$ & $\mathrm{p}(\mathrm{FDR})$ & $\mathrm{t}(20)$ & $\mathrm{p}(\mathrm{FDR})$ \\
\hline \multirow[t]{7}{*}{ R lateral parietal ctx. (DMN) } & R supramarginal g. (SAL) & 4.55 & 0.0085 & 3.67 & 0.0313 & 3.50 & 0.0057 \\
\hline & L supramarginal g. (SAL) & 3.68 & 0.017 & 3.65 & 0.0313 & 3.12 & 0.0108 \\
\hline & R inferior parietal s. (DMN) & N.S. & 3.65 & & 0.0313 & & N.S. \\
\hline & $\mathrm{R}$ lateral visual ctx. & 3.45 & 0.0194 & & N.S. & & N.S. \\
\hline & L lateral visual ctx. & 4.01 & 0.0152 & & N.S. & & N.S. \\
\hline & $\mathrm{R}$ inferior frontal $\mathrm{g}$. & 3.43 & 0.0194 & & N.S. & & N.S. \\
\hline & $\mathrm{L}$ posterior superior temporal $\mathrm{g}$. & 3.66 & 0.017 & & N.S. & & N.S. \\
\hline \multirow[t]{2}{*}{ L posterior parietal ctx. (FPN) } & $\mathrm{R}$ nucleus accumbens & 5.35 & 0.0014 & & N.S. & & N.S. \\
\hline & L nucleus accumbens & 4.25 & 0.0171 & & N.S. & & N.S. \\
\hline
\end{tabular}

N.S. not significant at $p_{F D R} \leq 0.05$. ctx cortex, $g$. gyrus, inf. inferior, sup. superior, post. posterior, $n$. nucleus, $S A L$ salience network, $F D R$ false-discovery rate correction as represented in Fig. 1a. FDR correction by Benjamini-Hochberg method

a reduction in uncorrelated variations within the regional signals. Although we have referred to these "uncorrelated variations" as "noise," they may represent unrelated neuronal information (Wens et al. 2019), neurovascular effects or cerebral metabolic rate (Kim et al. 2013), or random noise. We should also note that some differences in spectral power were observed between practitioners and controls at baseline, and between controls at follow-up and controls at baseline, which likely reflect true stochastic or random variations and indicate that our sample was underpowered. It is therefore possible that significant differences in regional coherence could be elicited from a larger cohort.

In addition to changes to the triple network that we hypothesized would be impacted by mindfulness practice, we also observed increased connectivity between the posterior parietal cortex (CEN) and the bilateral nucleus accumbens. Less is known about the role of the nucleus accumbens in mindfulness training, but a previous study

Table 3 Results of spectral density analyses: $t$ tests by treatment and scan (baseline or follow-up)

\begin{tabular}{|c|c|c|c|c|c|c|}
\hline Region of interest & Role & Comparison & & freq $(\mathrm{mHz})$ & $t$ & $p$ \\
\hline \multirow[t]{7}{*}{$\mathrm{R}$ lateral parietal ctx. } & \multirow[t]{7}{*}{ Network hub } & Controls at follow-up & $>$ Controls at baseline & 9.58 & -2.4496 & 0.0314 \\
\hline & & \multirow[t]{4}{*}{ Practitioners at follow-up } & \multirow[t]{4}{*}{$>$ Practitioners at baseline } & 0.21 & +2.5469 & 0.0235 \\
\hline & & & & 5.21 & -2.2669 & 0.0344 \\
\hline & & & & 7.71 & +2.1077 & 0.0496 \\
\hline & & & & 11.46 & -2.3487 & 0.0321 \\
\hline & & Practitioners at baseline & $>$ Controls at baseline & 9.58 & +2.5579 & 0.0272 \\
\hline & & Practitioners at follow-up & $>$ Controls at follow-up & Not significant & & \\
\hline \multirow[t]{7}{*}{ L supramarginal g. } & \multirow[t]{7}{*}{ Vertex } & Controls at follow-up & $>$ Controls at baseline & Not significant & & \\
\hline & & Practitioners at baseline & $>$ Controls at baseline & 8.33 & -2.2392 & 0.0393 \\
\hline & & & & 9.79 & -2.0850 & 0.0497 \\
\hline & & Practitioners at baseline & $>$ Controls at baseline & 4.58 & -2.4883 & 0.0252 \\
\hline & & & & 5.42 & -2.2572 & 0.0356 \\
\hline & & & & 10.00 & -2.2853 & 0.0344 \\
\hline & & Practitioners at follow-up & $>$ Controls at follow-up & Not significant & & \\
\hline \multirow[t]{4}{*}{ R supramarginal g. } & \multirow[t]{4}{*}{ Vertex } & Controls at follow-up & $>$ Controls at baseline & 1.04 & +2.2975 & 0.0460 \\
\hline & & Practitioners at follow-up & $>$ Practitioners at baseline & 9.17 & -2.1101 & 0.0495 \\
\hline & & Practitioners at baseline & $>$ Controls at baseline & 1.04 & -2.2534 & 0.0368 \\
\hline & & Practitioners at follow-up & $>$ Controls at follow-up & Not significant & & \\
\hline
\end{tabular}

STG superior temporal gyrus, freq $(\mathrm{mHz})$ frequency in millihertz. Only differences significant at $p \leq 0.05$ are shown 
(a)

CONTROLS

PRACTITIONERS
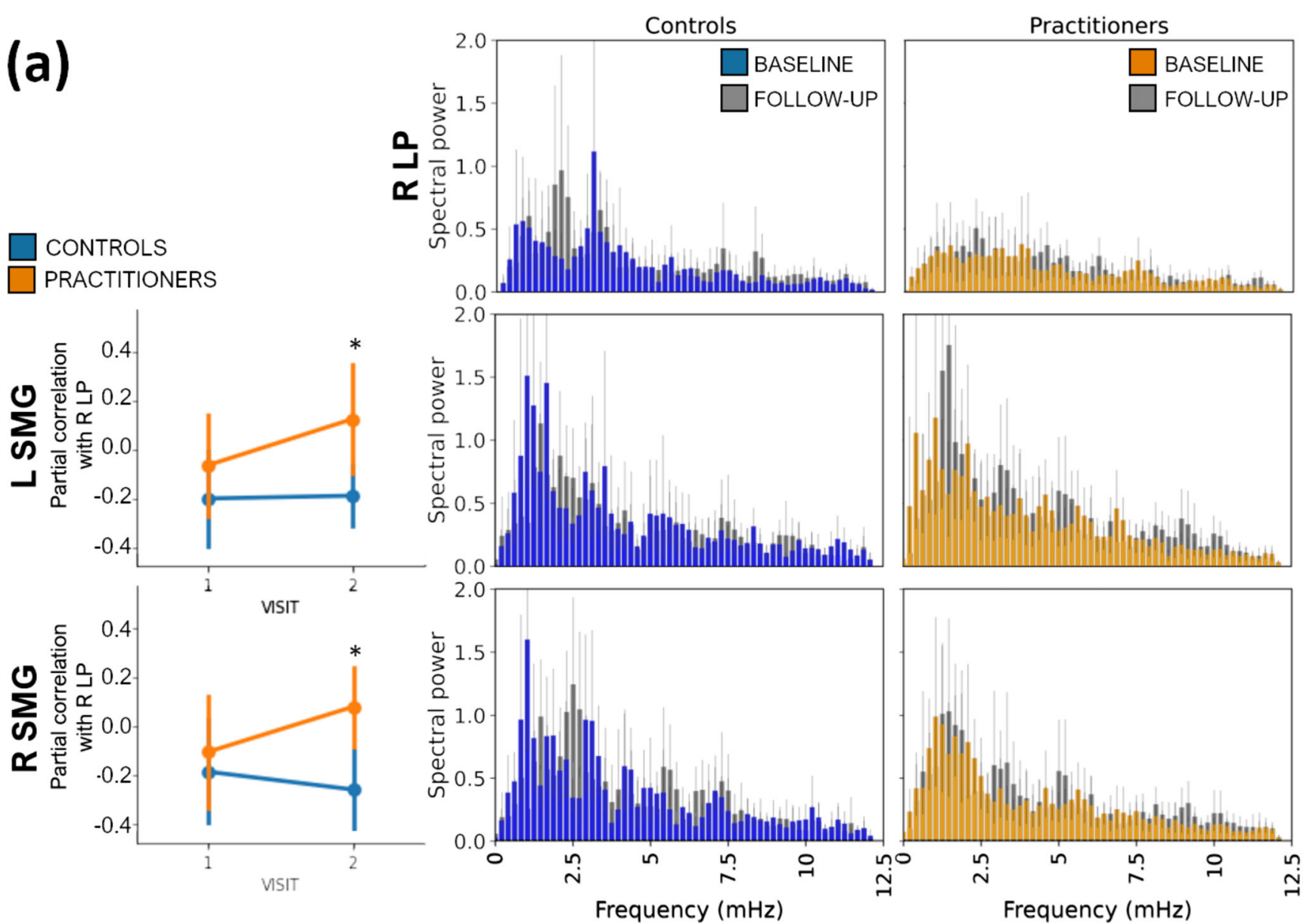

(b)
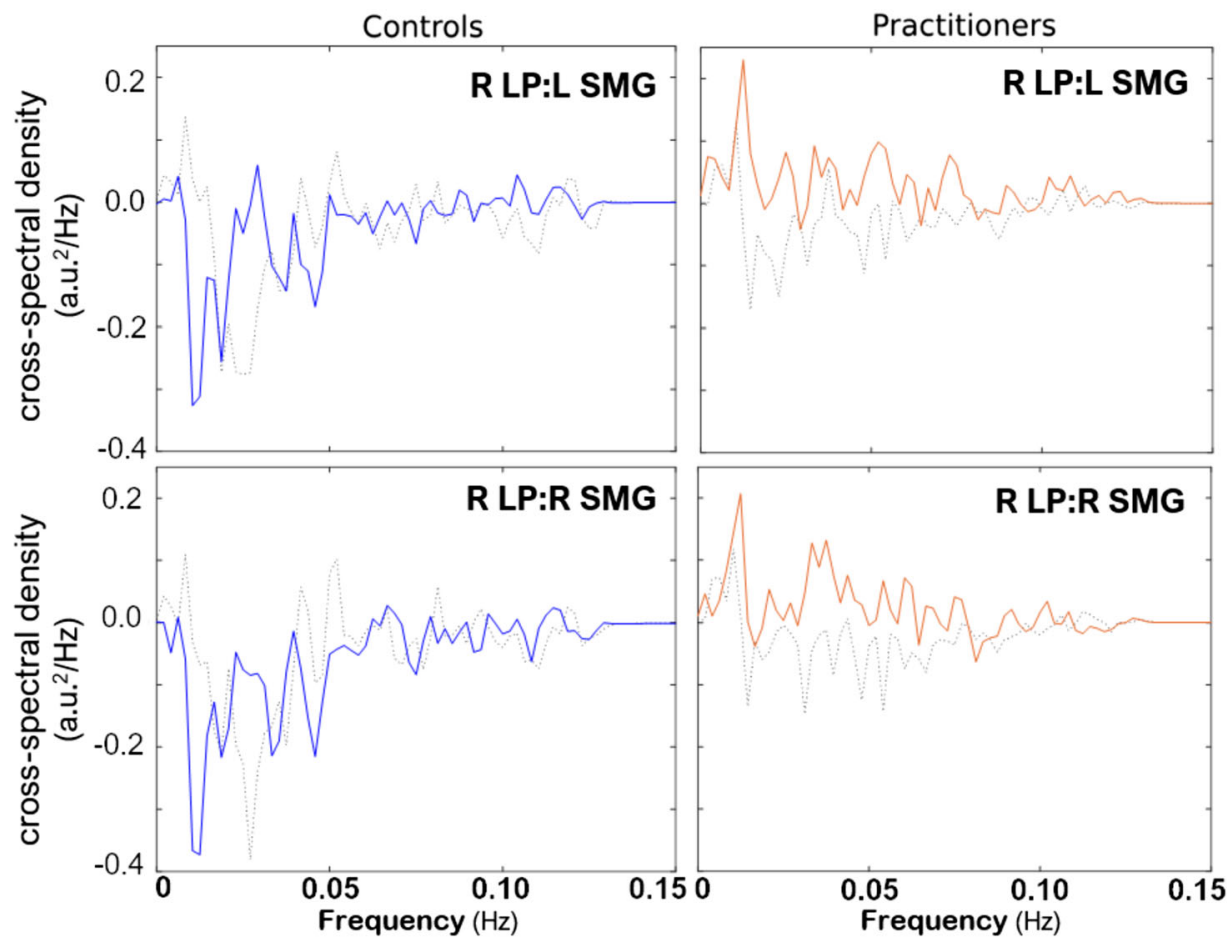
Fig. 4 a Comparison of regional changes in connectivity with the right hemisphere lateral parietal cortex (left column) and changes in spectral power (middle and right columns). Practitioners and controls are represented with amber and blue, respectively. $p_{\mathrm{FDR}} \leq 0.05$. b Crossspectral density (shared spectral power) between right lateral parietal cortex and left supramarginal (left column) and right supramarginal gyrus (right column) in controls (blue) and practitioners (amber) at +8 weeks. Baseline cross-spectral densities are presented as dotted lines

found that trait mindfulness scores were inversely correlated with gray matter volume in the bilateral accumbens (Taren et al. 2013). Some have theorized that mindfulness, especially in the context of reappraisal and emotion regulation, may augment functional coupling between networks important for executive control and motivation systems, including the nucleus accumbens (McConnell and Froeliger 2015). This account is supported by research linking dispositional mindfulness with inhibitory control (Oberle et al. 2012) and by the large body of evidence supporting the efficacy of mindfulness-based interventions for reward processing in the context of addiction (Froeliger et al. 2017; Garland and Howard 2018). The finding here may indicate that mindfulness training increased top-down executive control over bottom-up regions involved in motivation. Coupling a whole-brain connectome approach with strict corrections for multiple comparisons allowed us to interrogate the entire brain in an exploratory approach that we believe has the potential to identify changes in connectivity beyond the triple network.

This study adds to what is known about the effects of app-delivered mindfulness meditation. The feasibility and apparent effectiveness of app-delivered mindfulness meditation for healthcare trainees are particularly promising given the apparent Catch-22 with contemplative practices, which is that meditation-based health benefits may be most helpful for populations under high levels of stress and distress (Creswell and Lindsay 2014). Currently, the most studied and utilized mindfulness program, MBSR, traditionally consists of 8-10 classes lasting $2.5 \mathrm{~h}$ and an allday retreat, totaling approximately $26 \mathrm{~h}$ of session time. Carmody and Baer note that the lengthy format has proven prohibitive in several clinical trials even outside the context of health professions and provider well-being (Carmody and Baer 2009), and MBSR is likely even more prohibitive for health professions trainees already facing intense time demands. Recent commentaries call for trainee and provider wellness programs that are sustainable and preventive in nature rather than reactive (Chaukos et al. 2018; Slavin 2016; Wasson et al. 2016), and the results presented here indicate that brief app-delivered mindfulness training has demonstrable effects on brain function and self-reported mindfulness. Smartphone app-delivered mindfulness has received copious attention with an increase in the number and variety of available apps, the number of people using the apps, and in both number and quality of research studies on app-based interventions (Cox et al. 2019; Linardon 2020; Walsh et al. 2019). While the findings reported here are broadly consistent with that growing literature in finding that app-delivered mindfulness affects outcomes of interest, caution toward app-delivered mindfulness is warranted for a number of reasons. First, the majority of studies to date, including ours, have examined relatively short-term outcomes, and the long-term impact of mindfulness appbased interventions is not clear (Linardon 2020). Moreover, several studies indicate that acceptability and participant interest impact the extent to which study participants engage with app content (Howells et al. 2016; Mascaro et al. 2020) and many studies of smartphone app-delivered interventions report high levels of attrition (e.g., Linardon and Fuller-Tyszkiewicz 2020; Torous et al. 2020). This study used an intent-to-treat design, which included 2 study participants who never downloaded the app, and future research to understand the barriers and facilitators to app use among trainees will be important in this area of meditation research. Crucially, there is a standing question as to whether app-delivered mindfulness contains the key elements necessary to qualify as a mindfulness intervention. By some accounts, the relational didactic interaction between the participant and an instructor is an essential feature of a mindfulness-based program (Crane et al. 2017). Moreover, as apps proliferate and expand both the number and diversity of instructors represented within the apps as well as the instructional content delivered, there is an inherent trade-off: app-delivered mindfulness will likely become more flexible and widely acceptable, but also more difficult to study.

The fact that the findings were driven by PA students likely reflects that we were underpowered to detect changes in the surgery residents. The residents and PA students did not differ prior to randomization on any of the mindfulness subscales, nor did they differ in practice time. Moreover, previous research found that mindfulness training was effective for surgery residents and impacted brain function (Lebares et al. 2019). However, it remains possible that the app was more effective for PA students and more research is critical for understanding the mental health and wellness needs of PA students, which may be different than the needs of other trainees (Hernandez et al. 2010). In sum, a thorough evaluation of these effects, and their potential benefit to trainees in the health professions, would require a randomized, controlled clinical trial-type 
design, for which the present study provides only preliminary data.

\section{Limitations and Future Research Directions}

While the current study had several notable strengths and contributes to our understanding of how mindfulness meditation impacts brain function, the study had some limitations that warrant discussion. Recent research using high resolution MRI and analytic methods sensitive to individual variation indicates that the DMN may be best understood as two or more separate networks that are located in close proximity but functionally distinct (Braga et al. 2019; Kernbach et al. 2018; Kong et al. 2018). However, examining this level of detail is difficult within a group-averaged functional connectivity analysis. In addition, this was a very small sample and future studies with a larger and more diverse population of health profession trainees will be important. Related, future studies should begin to compare multiple active conditions or interventional approaches, with the awareness that trainees have extreme time constraints and will be limited in the time they can devote to activities outside their training demands. While we believe these findings add to what is known about interventional approaches to well-being among trainees, connectivity changes do not necessarily translate to meaningful or lasting improvements to trainee mental health and scientific humility will be important toward not over-ascribing the importance of statistically significant neuroimaging findings (Tang et al. 2015; Van Dam et al. 2018). The methods used here identified changes in connectivity related to app-delivered mindfulness training, and future research using these methods can more definitively interrogate whether connectivity changes lead to the changes in well-being so important for health profession trainees.

Acknowledgments We would like to express our gratitude to research assistants who helped collect the data reported here and to faculty in the Emory PA program for facilitating student recruitment. We are also grateful to Chris Larsen, MD, DPhil for his guidance and expertise in embarking on this research and to $10 \%$ Happier for providing app subscriptions. $10 \%$ Happier provided input on recommended meditation content during study design, but did not have access to study data and was not involved in data analysis or interpretation. No member of the study team has any commercial interest in $10 \%$ Happier.

Authors' Contributions JLS analyzed the fMRI data and collaborated in writing the paper. JWA collaborated with analyzing the fMRI data and writing the paper. $\mathrm{CH}$ collaborated in designing the study and editing the final manuscript. KW executed the study and collaborated in editing the final manuscript. KA collaborated with analyzing the data and editing the final manuscript. MBL collaborated in designing the study and editing the final manuscript. JSM conceived of the study, executed the data collection, and collaborated in analyzing the data and writing the paper.

\section{Compliance with Ethical Standards}

Conflict of Interest The authors declare that they have no conflict of interest.

Ethical Approval The present study was approved by the Emory University Institutional Review Board (IRB00088349). All procedures were conducted in accordance with the ethical standards of the IRB and with the 1964 Helsinki declaration and comparable ethical standards.

Informed Consent Written informed consent was obtained from all participants prior to participation in the study.

\section{References}

Ashburner, J. (2007). A fast diffeomorphic image registration algorithm. Neuroimage, 38(1), 95-113.

Ashburner, J., \& Friston, K. J. (2011). Diffeomorphic registration using geodesic shooting and Gauss-Newton optimisation. Neuroimage, 55(3), 954-967.

Baer, R. A., Smith, G. T., \& Allen, K. B. (2004). Assessment of mindfulness by self-report: the Kentucky inventory of mindfulness skills. Assessment, 11(3), 191-206.

Baer, R. A., Smith, G. T., Lykins, E., Button, D., Krietemeyer, J., Sauer, S., Walsh, E., Duggan, D., \& Williams, J. M. G. (2008). Construct validity of the five facet mindfulness questionnaire in meditating and nonmeditating samples. Assessment, 15(3), 329-342.

Behzadi, Y., Restom, K., Liau, J., \& Liu, T. T. (2007). A component based noise correction method (CompCor) for BOLD and perfusion based fMRI. Neuroimage, 37(1), 90-101.

Benjamini, Y., \& Hochberg, Y. (1995). Controlling the false discovery rate: a practical and powerful approach to multiple testing. Journal of the Royal Statistical Society: Series B: Methodological, 57(1), 289-300.

Bilevicius, E., Smith, S. D., \& Kornelsen, J. (2018). Resting-state network functional connectivity patterns associated with the mindful attention awareness scale. Brain Connectivity, 8(1), 40-48.

Birn, R. M., Diamond, J. B., Smith, M. A., \& Bandettini, P. A. (2006). Separating respiratory-variation-related fluctuations from neuronalactivity-related fluctuations in fMRI. Neuroimage, 31(4), 15361548.

Black, D. S., \& Slavich, G. M. (2016). Mindfulness meditation and the immune system: a systematic review of randomized controlled trials. Annals of the New York Academy of Sciences, 1373(1), 13.

Braga, R. M., Van Dijk, K. R., Polimeni, J. R., Eldaief, M. C., \& Buckner, R. L. (2019). Parallel distributed networks resolved at high resolution reveal close juxtaposition of distinct regions. Journal of Neurophysiology, 121(4), 1513-1534.

Brewer, J. A., Worhunsky, P. D., Gray, J. R., Tang, Y.-Y., Weber, J., \& Kober, H. (2011). Meditation experience is associated with differences in default mode network activity and connectivity. Proceedings of the National Academy of Sciences, 108(50), 20254-20259.

Buckner, R. L., \& DiNicola, L. M. (2019). The brain's default network: updated anatomy, physiology and evolving insights. Nature Reviews Neuroscience, 20(10), 593-608.

Carmody, J., \& Baer, R. A. (2009). How long does a mindfulness-based stress reduction program need to be? A review of class contact hours and effect sizes for psychological distress. Journal of Clinical Psychology, 65(6), 627-638.

Chai, X. J., Castañón, A. N., Öngür, D., \& Whitfield-Gabrieli, S. (2012). Anticorrelations in resting state networks without global signal regression. Neuroimage, 59(2), 1420-1428. 
Chaukos, D., Chad-Friedman, E., Mehta, D. H., Byerly, L., Celik, A., McCoy, T. H., \& Denninger, J. W. (2017). Risk and resilience factors associated with resident burnout. Academic Psychiatry, 41(2), 189-194.

Chaukos, D., Vestal, H. S., Bernstein, C. A., Belitsky, R., Cohen, M. J., Hutner, L., Penzner, J., Scheiber, S., Wrzosek, M. I., \& Silberman, E. K. (2018). An ounce of prevention: a public health approach to improving physician well-being. Academic Psychiatry, 42(1), 150154.

Cocke, K. D., Klocko, D. J., \& Kindratt, T. B. (2019). Screening for undetected depression in physician assistant students. The Journal of Physician Assistant Education, 30(2), 118-121.

Cox, C. E., Hough, C. L., Jones, D. M., Ungar, A., Reagan, W., Key, M. D., Gremore, T., Olsen, M. K., Sanders, L., \& Greeson, J. M. (2019). Effects of mindfulness training programmes delivered by a selfdirected mobile app and by telephone compared with an education programme for survivors of critical illness: a pilot randomised clinical trial. Thorax, 74(1), 33-42.

Crane, R. S., Brewer, J., Feldman, C., Kabat-Zinn, J., Santorelli, S., Williams, J. M. G., \& Kuyken, W. (2017). What defines mindfulness-based programs? The warp and the weft. Psychological Medicine, 47(6), 990-999.

Creswell, J. D., \& Lindsay, E. K. (2014). How does mindfulness training affect health? A mindfulness stress buffering account. Current Directions in Psychological Science, 23(6), 401-407.

Creswell, J. D., Taren, A. A., Lindsay, E. K., Greco, C. M., Gianaros, P. J., Fairgrieve, A., Marsland, A. L., Brown, K. W., Way, B. M., \& Rosen, R. K. (2016). Alterations in resting-state functional connectivity link mindfulness meditation with reduced interleukin-6: a randomized controlled trial. Biological Psychiatry, 80(1), 53-61.

Demarzo, M. M., Montero-Marin, J., Cuijpers, P., Zabaleta-del-Olmo, E., Mahtani, K. R., Vellinga, A., Vicens, C., López-del-Hoyo, Y., \& García-Campayo, J. (2015). The efficacy of mindfulness-based interventions in primary care: a meta-analytic review. The Annals of Family Medicine, 13(6), 573-582.

Desikan, R. S., Ségonne, F., Fischl, B., Quinn, B. T., Dickerson, B. C., Blacker, D., Buckner, R. L., Dale, A. M., Maguire, R. P., \& Hyman, B. T. (2006). An automated labeling system for subdividing the human cerebral cortex on MRI scans into gyral based regions of interest. Neuroimage, 31(3), 968-980.

Dickenson, J., Berkman, E. T., Arch, J., \& Lieberman, M. D. (2012). Neural correlates of focused attention during a brief mindfulness induction. Social Cognitive and Affective Neuroscience, 8(1), 4047.

Dobkin, P. L., \& Hutchinson, T. A. (2013). Teaching mindfulness in medical school: where are we now and where are we going? Medical Education, 47(8), 768-779.

Doll, A., Hölzel, B. K., Boucard, C. C., Wohlschläger, A. M., \& Sorg, C. (2015). Mindfulness is associated with intrinsic functional connectivity between default mode and salience networks. Frontiers in Human Neuroscience, 9, 461.

Doll, A., Hölzel, B. K., Bratec, S. M., Boucard, C. C., Xie, X., Wohlschläger, A. M., \& Sorg, C. (2016). Mindful attention to breath regulates emotions via increased amygdala-prefrontal cortex connectivity. Neuroimage, 134, 305-313.

Downar, J., Crawley, A. P., Mikulis, D. J., \& Davis, K. D. (2002). A cortical network sensitive to stimulus salience in a neutral behavioral context across multiple sensory modalities. Journal of Neurophysiology, 87(1), 615-620.

Duff, E. P., Johnston, L. A., Xiong, J., Fox, P. T., Mareels, I., \& Egan, G. F. (2008). The power of spectral density analysis for mapping endogenous BOLD signal fluctuations. Human Brain Mapping, 29(7), 778-790.

Economides, M., Martman, J., Bell, M. J., \& Sanderson, B. (2018). Improvements in stress, affect, and irritability following brief use of a mindfulness-based smartphone app: a randomized controlled trial. Mindfulness, 9(5), 1584-1593.

Farb, N. A., Segal, Z. V., Mayberg, H., Bean, J., McKeon, D., Fatima, Z., \& Anderson, A. K. (2007). Attending to the present: mindfulness meditation reveals distinct neural modes of self-reference. Social Cognitive and Affective Neuroscience, 2(4), 313-322.

Farb, N. A., Segal, Z. V., \& Anderson, A. K. (2012). Mindfulness meditation training alters cortical representations of interoceptive attention. Social Cognitive and Affective Neuroscience, 8(1), 15-26.

Fox, M. D., Snyder, A. Z., McAvoy, M. P., Barch, D. M., \& Raichle, M. E. (2005). The BOLD onset transient: identification of novel functional differences in schizophrenia. Neuroimage, 25(3), 771-782.

Fox, M. D., Zhang, D., Snyder, A. Z., \& Raichle, M. E. (2009). The global signal and observed anticorrelated resting state brain networks. Journal of Neurophysiology, 101(6), 3270-3283.

Fransson, P. (2006). How default is the default mode of brain function? Further evidence from intrinsic BOLD signal fluctuations. Neuropsychologia, 44(14), 2836-2845.

Frazier, J. A., Chiu, S., Breeze, J. L., Makris, N., Lange, N., Kennedy, D. N., Herbert, M. R., Bent, E. K., Koneru, V. K., \& Dieterich, M. E. (2005). Structural brain magnetic resonance imaging of limbic and thalamic volumes in pediatric bipolar disorder. American Journal of Psychiatry, 162(7), 1256-1265.

Froeliger, B., Mathew, A. R., McConnell, P., Eichberg, C., Saladin, M., Carpenter, M., \& Garland, E. (2017). Restructuring reward mechanisms in nicotine addiction: a pilot fMRI study of mindfulnessoriented recovery enhancement for cigarette smokers. EvidenceBased Complementary and Alternative Medicine, eCAM, 2017, Article 7018014. https://doi.org/10.1155/2017/7018014.

Garland, E. L., \& Howard, M. O. (2018). Mindfulness-based treatment of addiction: current state of the field and envisioning the next wave of research. Addiction Science \& Clinical Practice, 13(1), 14.

Gotink, R. A., Meijboom, R., Vernooij, M. W., Smits, M., \& Hunink, M. M. (2016). 8-week mindfulness based stress reduction induces brain changes similar to traditional long-term meditation practice-a systematic review. Brain and Cognition, 108, 32-41.

Goyal, M., Singh, S., Sibinga, E. S., et al. (2014). Meditation programs for psychological stress and well-being: a systematic review and meta-analysis. JAMA Internal Medicine, 174(3), 357-368.

Haase, L., May, A. C., Falahpour, M., Isakovic, S., Simmons, A. N., Hickman, S. D., Liu, T. T., \& Paulus, M. P. (2015). A pilot study investigating changes in neural processing after mindfulness training in elite athletes. Frontiers in Behavioral Neuroscience, 9, 229.

Harris, D., Warren, J., \& Adler, C. (2018). Meditation for fidgety skeptics: a 10\% happier how-to book. Spiegel \& Grau.

Hasenkamp, W., \& Barsalou, L. W. (2012). Effects of meditation experience on functional connectivity of distributed brain networks. Frontiers in Human Neuroscience, 6, 38.

Hasenkamp, W., Wilson-Mendenhall, C. D., Duncan, E., \& Barsalou, L. W. (2012). Mind wandering and attention during focused meditation: a fine-grained temporal analysis of fluctuating cognitive states. Neuroimage, 59(1), 750-760.

Hernandez, M. B., Blavo, C., Hardigan, P. C., Perez, A. M., \& Hage, K. (2010). Differences in perceived stress, depression, and medical symptoms among medical, nursing, and physician assistant students: a latent class analysis. Annals of Behavioral Science and Medical Education, 16(1), 35-39.

Hölzel, B. K., Hoge, E. A., Greve, D. N., Gard, T., Creswell, J. D., Brown, K. W., Barrett, L. F., Schwartz, C., Vaitl, D., \& Lazar, S. W. (2013). Neural mechanisms of symptom improvements in generalized anxiety disorder following mindfulness training. NeuroImage: Clinical, 2, 448-458.

Howells, A., Ivtzan, I., \& Eiroa-Orosa, F. J. (2016). Putting the 'app' in happiness: a randomised controlled trial of a smartphone-based mindfulness intervention to enhance wellbeing [journal article]. Journal of Happiness Studies, 17(1), 163-185. 
Jafri, M. J., Pearlson, G. D., Stevens, M., \& Calhoun, V. D. (2008). A method for functional network connectivity among spatially independent resting-state components in schizophrenia. Neuroimage, 39(4), 1666-1681.

Jang, J. H., Jung, W. H., Kang, D.-H., Byun, M. S., Kwon, S. J., Choi, C.H., \& Kwon, J. S. (2011). Increased default mode network connectivity associated with meditation. Neuroscience Letters, 487(3), 358-362.

Kelly, A. C., Uddin, L. Q., Biswal, B. B., Castellanos, F. X., \& Milham, M. P. (2008). Competition between functional brain networks mediates behavioral variability. Neuroimage, 39(1), 527-537.

Kernbach, J. M., Yeo, B. T., Smallwood, J., Margulies, D. S., de Schotten, M. T., Walter, H., Sabuncu, M. R., Holmes, A. J., Gramfort, A., \& Varoquaux, G. (2018). Subspecialization within default mode nodes characterized in 10,000 UK Biobank participants. Proceedings of the National Academy of Sciences, 115(48), 12295-12300.

Kilpatrick, L. A., Suyenobu, B. Y., Smith, S. R., Bueller, J. A., Goodman, T., Creswell, J. D., Tillisch, K., Mayer, E. A., \& Naliboff, B. D. (2011). Impact of mindfulness-based stress reduction training on intrinsic brain connectivity. Neuroimage, 56(1), 290-298.

Kim, J. Y., Kim, S. H., Seo, J., Kim, S. H., Han, S. W., Nam, E. J., Kim, S. K., Lee, H. J., Lee, S. J., Kim, Y. T., \& Chang, Y. (2013). Increased power spectral density in resting-state pain-related brain networks in fibromyalgia. Pain, 154(9), 1792-1797.

Koechlin, E., \& Summerfield, C. (2007). An information theoretical approach to prefrontal executive function. Trends in Cognitive Sciences, 11(6), 229-235.

Kong, R., Li, J., Orban, C., Sabuncu, M. R., Liu, H., Schaefer, A., Sun, N., Zuo, X.-N., Holmes, A. J., \& Eickhoff, S. B. (2018). Spatial topography of individual-specific cortical networks predicts human cognition, personality, and emotion. Cerebral Cortex, 29(6), 25332551.

Lebares, C. C., Hershberger, A. O., Guvva, E. V., Desai, A., Mitchell, J., Shen, W., Reilly, L. M., Delucchi, K. L., O'Sullivan, P. S., \& Ascher, N. L. (2018). Feasibility of formal mindfulness-based stress-resilience training among surgery interns: a randomized clinical trial. JAMA Surgery, 153(10), e182734-e182734.

Lebares, C. C., Guvva, E. V., Olaru, M., Sugrue, L. P., Staffaroni, A. M., Delucchi, K. L., Kramer, J. H., Ascher, N. L., \& Harris, H. W. (2019). Efficacy of mindfulness-based cognitive training in surgery: additional analysis of the mindful surgeon pilot randomized clinical trial. JAMA Network Open, 2(5), e194108.

Linardon, J. (2020). Can acceptance, mindfulness, and self-compassion be learned by smartphone apps? A systematic and meta-analytic review of randomized controlled trials. Behavior Therapy, 51(4), 646-658.

Linardon, J., \& Fuller-Tyszkiewicz, M. (2020). Attrition and adherence in smartphone-delivered interventions for mental health problems: a systematic and meta-analytic review. Journal of Consulting and Clinical Psychology, 88(1), 1-13.

Lindsay, E. K., Young, S., Smyth, J. M., Brown, K. W., \& Creswell, J. D. (2018). Acceptance lowers stress reactivity: dismantling mindfulness training in a randomized controlled trial. Psychoneuroendocrinology, $87,63-73$.

Lomas, T., Medina, J. C., Ivtzan, I., Rupprecht, S., \& Eiroa-Orosa, F. J. (2018). A systematic review and meta-analysis of the impact of mindfulness-based interventions on the well-being of healthcare professionals. Mindfulness, 10(7), 1193-1216.

Lutz, J., Herwig, U., Opialla, S., Hittmeyer, A., Jäncke, L., Rufer, M., Grosse Holtforth, M., \& Brühl, A. B. (2013). Mindfulness and emotion regulation - an fMRI study. Social Cognitive and Affective Neuroscience, 9(6), 776-785.

Lutz, A., Jha, A. P., Dunne, J. D., \& Saron, C. D. (2015). Investigating the phenomenological matrix of mindfulness-related practices from a neurocognitive perspective. American Psychologist, 70(7), 632-658.
Makris, N., Goldstein, J. M., Kennedy, D., Hodge, S. M., Caviness, V. S., Faraone, S. V., Tsuang, M. T., \& Seidman, L. J. (2006). Decreased volume of left and total anterior insular lobule in schizophrenia. Schizophrenia Research, 83(2-3), 155-171.

Mascaro, J. S., Wehrmeyer, K., Mahathre, V., \& Darcher, A. (2020). A longitudinal, randomized and controlled study of app-delivered mindfulness in the workplace. Journal of Wellness, 2(1), 4.

Mata, D. A., Ramos, M. A., Bansal, N., Khan, R., Guille, C., Di Angelantonio, E., \& Sen, S. (2015). Prevalence of depression and depressive symptoms among resident physicians: a systematic review and meta-analysis. JAMA, 314(22), 2373-2383.

McConnell, P. A., \& Froeliger, B. (2015). Mindfulness, mechanisms and meaning: perspectives from the cognitive neuroscience of addiction. Psychological Inquiry, 26(4), 349-357.

McConville, J., McAleer, R., \& Hahne, A. (2017). Mindfulness training for health profession students - The effect of mindfulness training on psychological well-being, learning and clinical performance of health professional students: a systematic review of randomized and non-randomized controlled trials. Explore, 13(1), 26-45.

Menon, V., \& Uddin, L. Q. (2010). Saliency, switching, attention and control: a network model of insula function. Brain Structure and Function, 214(5-6), 655-667.

Modinos, G., Ormel, J., \& Aleman, A. (2010). Individual differences in dispositional mindfulness and brain activity involved in reappraisal of emotion. Social Cognitive and Affective Neuroscience, 5(4), 369377.

Mooneyham, B. W., Mrazek, M. D., Mrazek, A. J., \& Schooler, J. W. (2016). Signal or noise: brain network interactions underlying the experience and training of mindfulness. Annals of the New York Academy of Sciences, 1369(1), 240-256.

Murakami, H., Nakao, T., Matsunaga, M., Kasuya, Y., Shinoda, J., Yamada, J., \& Ohira, H. (2012). The structure of mindful brain. PLoS One, 7(9), e46377.

Neuendorf, R., Wahbeh, H., Chamine, I., Yu, J., Hutchison, K., \& Oken, B. S. (2015). The effects of mind-body interventions on sleep quality: a systematic review. Evidence-based Complementary and Alternative Medicine: Ecam, 2015, 902708.

Oberle, E., Schonert-Reichl, K. A., Lawlor, M. S., \& Thomson, K. C. (2012). Mindfulness and inhibitory control in early adolescence. The Journal of Early Adolescence, 32(4), 565-588.

Ospina, M. B., Bond, K., Karkhaneh, M., Tjosvold, L., Vandermeer, B., Liang, Y., Bialy, L., Hooton, N., Buscemi, N., Dryden, D. M., \& Klassen, T. P. (2008). Meditation practices for health: state of the research. Evidence Report/Technology Assessment, 155, 1-263.

Petrides, M. (2005). Lateral prefrontal cortex: architectonic and functional organization. Philosophical Transactions of the Royal Society, B: Biological Sciences, 360(1456), 781-795.

Prakash, R., Rastogi, P., Dubey, I., Abhishek, P., Chaudhury, S., \& Small, B. J. (2012). Long-term concentrative meditation and cognitive performance among older adults. Neuropsychology, Development, and Cognition. Section B, Aging, Neuropsychology and Cognition, 19(4), 479-494.

Rotenstein, L. S., Ramos, M. A., Torre, M., Segal, J. B., Peluso, M. J., Guille, C., Sen, S., \& Mata, D. A. (2016). Prevalence of depression, depressive symptoms, and suicidal ideation among medical students: a systematic review and meta-analysis. JAMA, 316(21), 2214-2236.

Seeley, W. W., Menon, V., Schatzberg, A. F., Keller, J., Glover, G. H., Kenna, H., Reiss, A. L., \& Greicius, M. D. (2007). Dissociable intrinsic connectivity networks for salience processing and executive control. The Journal of Neuroscience, 27(9), 2349-2356.

Slavin, S. J. (2016). Medical student mental health: culture, environment, and the need for change. JAMA, 316(21), 2195-2196.

Tang, Y.-Y., Hölzel, B. K., \& Posner, M. I. (2015). The neuroscience of mindfulness meditation. Nature Reviews Neuroscience, 16(4), 213 225 . 
Taren, A. A., Creswell, J. D., \& Gianaros, P. J. (2013). Dispositional mindfulness co-varies with smaller amygdala and caudate volumes in community adults. PLoS One, 8(5), e64574.

Taylor, V. A., Daneault, V., Grant, J., Scavone, G., Breton, E., RoffeVidal, S., Courtemanche, J., Lavarenne, A. S., Marrelec, G., \& Benali, H. (2012). Impact of meditation training on the default mode network during a restful state. Social Cognitive and Affective Neuroscience, 8(1), 4-14.

tenpercent.com. (2020). Retrieved June 26, 2020 from https://www. tenpercent.com/mindfulness-meditation-the-basics

Torous, J., Lipschitz, J., Ng, M., \& Firth, J. (2020). Dropout rates in clinical trials of smartphone apps for depressive symptoms: a systematic review and meta-analysis. Journal of Affective Disorders, 263, 413-419.

Tzourio-Mazoyer, N., Landeau, B., Papathanassiou, D., Crivello, F., Etard, O., Delcroix, N., Mazoyer, B., \& Joliot, M. (2002). Automated anatomical labeling of activations in SPM using a macroscopic anatomical parcellation of the MNI MRI single-subject brain. Neuroimage, 15(1), 273-289.

Van Dam, N. T., Hobkirk, A. L., Danoff-Burg, S., \& Earleywine, M. (2012). Mind your words: positive and negative items create method effects on the Five Facet Mindfulness Questionnaire. Assessment, 19(2), 198-204.

Van Dam, N. T., Van Vugt, M. K., Vago, D. R., Schmalzl, L., Saron, C. D., Olendzki, A., Meissner, T., Lazar, S. W., Kerr, C. E., \& Gorchov, J. (2018). Mind the hype: a critical evaluation and prescriptive agenda for research on mindfulness and meditation. Perspectives on Psychological Science, 13(1), 36-61.

Walsh, K. M., Saab, B. J., \& Farb, N. A. (2019). Effects of a mindfulness meditation app on subjective well-being: active randomized controlled trial and experience sampling study. JMIR Mental Health, 6(1), e10844.
Wang, L., Shen, H., Lei, Y., Zeng, L.-L., Cao, F., Su, L., Yang, Z., Yao, S., \& Hu, D. (2017). Altered default mode, fronto-parietal and salience networks in adolescents with Internet addiction. Addictive Behaviors, 70, 1-6.

Wasson, L. T., Cusmano, A., Meli, L., et al. (2016). Association between learning environment interventions and medical student well-being: A systematic review. JAMA, 316(21), 2237-2252.

Wells, R. E., Yeh, G. Y., Kerr, C. E., Wolkin, J., Davis, R. B., Tan, Y., Spaeth, R., Wall, R. B., Walsh, J., \& Kaptchuk, T. J. (2013). Meditation's impact on default mode network and hippocampus in mild cognitive impairment: a pilot study. Neuroscience Letters, 556, $15-19$.

Wens, V., Bourguignon, M., Vander Ghinst, M., Mary, A., Marty, B., Coquelet, N., Naeije, G., Peigneux, P., Goldman, S., \& De Tiège, X. (2019). Synchrony, metastability, dynamic integration, and competition in the spontaneous functional connectivity of the human brain. Neuroimage, 199, 313-324.

Whitfield-Gabrieli, S., \& Nieto-Castanon, A. (2012). Conn: a functional connectivity toolbox for correlated and anticorrelated brain networks. Brain Connectivity, 2(3), 125-141.

Worsley, K. J., Marrett, S., Neelin, P., Vandal, A. C., Friston, K. J., \& Evans, A. C. (1996). A unified statistical approach for determining significant signals in images of cerebral activation. Human Brain Mapping, 4(1), 58-73.

Wylde, C. M., Mahrer, N. E., Meyer, R. M., \& Gold, J. I. (2017). Mindfulness for novice pediatric nurses: Smartphone application versus traditional intervention. Journal of Pediatric Nursing: Nursing Care of Children and Families, 36, 205-212.

Publisher's Note Springer Nature remains neutral with regard to jurisdictional claims in published maps and institutional affiliations. 